\title{
Stadien der Veränderung: Modell, Anwendungsbewährung und Perspektiven im Suchtbereich
}

Jürgen Hoyer

\author{
Stages of Change: Model, Evaluation, and Perspectives in Substance Disorders
}

\section{Zusammenfassung}

Das Modell der „Stadien der Veränderung“ wird vorgestellt und sein Anwendungsnutzen für Forschung und Praxis im Suchtbereich kritisch evaluiert. Dabei wird deutlich, dass das Modell in seiner vorliegenden Form nur eine empirisch unbefriedigende globale Orientierung über Veränderungsschritte bei Suchtpatienten bietet. Spezifische Modelle, die Besonderheiten von Behandlungsgruppen und -settings berücksichtigen, scheinen Erfolg versprechender. Außerdem wird aufgrund neuer Befunde vorgeschlagen, im Suchtbereich zusätzliche „Stadien“ zu berücksichtigen: Rückfall, Rückfallgefahr und Resignation.

\section{Schlïisselwörter}

Stadien der Veränderung · Alkoholabhängigkeit · Drogenabhängigkeit · Rauchen · Suchttherapie · Rückfallstadium

\section{Abstract}

The stages of change approach is described and its use for research and practice is critically reviewed. The model in its present form allows for a global orientation concerning therapeutic change in addiction but has not been shown to be empirically convincing. Models which are more straightforwardly directed toward the specificity of treatment group and setting are expected to be more successful. Finally, based on new empirical results additional stages to describe addictive behaviours are proposed: relapse, relapse danger, and resignation/drop-out.

\section{Key words}

Stages of change - alcohol dependence $\cdot$ drug addiction $\cdot$ smoking · drug treatment $\cdot$ relapse stage

\section{Einleitung}

Eine klare Orientierung über den therapeutischen Veränderungsprozess ist nicht nur im Suchtbereich für Praktiker und Forscher von großer Bedeutung. Modellüberlegungen über die typische Abfolge von Phasen oder Teilschritten im Rahmen einer Psychotherapie gibt es deshalb in jeder Therapieschule (vgl. [1]). Am weitesten verbreitet ist das Konzept der „Stadien der Veränderung“ [2]. Inzwischen nicht weniger als 2796 Einträge im Suchsystem PsycInfo für das Schlüsselwort „stages of change“ (Stand Juli 2003) belegen dies.
Ziel des vorliegenden Beitrags ist es, das Modell und ausgewählte empirische Studien in einem kritischen Überblick darzustellen. Dabei werden konzeptuelle Schwächen und Probleme bei der Operationalisierung und empirischen Bewährung deutlich. Als Konsequenz daraus ergeben sich Vorschläge zur Modifikation des Modells im Suchtbereich.

Institutsangaben

Klinische Psychologie und Psychotherapie, Technische Universität Dresden

Korrespondenzadresse

Prof. Dr. Jürgen Hoyer · Technische Universität Dresden

Institutsambulanz und Tagesklinik für Psychotherapie · Hohe Str. 53 · 01087 Dresden ·

E-mail: hoyer@psychologie.tu-dresden.de

Bibliografie

Suchttherapie 2003; 4: 140-145 @ Georg Thieme Verlag Stuttgart · New York · ISSN 1439-9903 
Die Annahmen über „Stadien der Veränderung“ sind ein Teil des so genannten „Transtheoretischen Modells“, mit dem Prochaska, DiClemente und Norcross [2] eine umfassende und therapieschulenunabhängige Beschreibung der Psychotherapie einschließlich typischer Verläufe und Prozesse anstreben. Sie postulieren für die psychologischen - insbesondere für die motivationalen - Prozesse bei der Veränderung problematischer Verhaltensweisen folgende fünf Stadien:

- ein vorintentionales Stadium (Precontemplation), in dem eine Verhaltensänderung nicht in Erwägung gezogen wird;

- ein Stadium der Absichtsbildung (Contemplation), in dem eine Verhaltensänderung ernsthaft erwogen wird;

- ein Vorbereitungsstadium (Preparation), in dem die Absicht zur Verhaltensänderung besteht und dessen Voraussetzungen hergestellt werden;

- ein Handlungsstadium (Action), das die aktiven Versuche der Veränderung beinhaltet; und

- ein Aufrechterhaltungsstadium (Maintenance), das durch die (aktive) Beibehaltung einer positiven Verhaltensänderung gekennzeichnet ist (nähere Darstellung vgl. [3]).

Später wurde ein Beendigungsstadium (Termination) ergänzt. ${ }^{1}$

Der Ausstiegsprozess aus einer Abhängigkeit (oder anderen schädigenden Gewohnheiten) muss dabei nicht durch ein kontinuierliches Fortschreiten über diese Stadien gekennzeichnet sein; es kann auch zu einem oder mehrfachen Rückschritten oder zu mehrmaligem Durchlaufen der Abfolge kommen [2].

Es wird angenommen, dass die Stadien distinkt sind und dass sie sich durch eine unterschiedliche Motivationslage im Hinblick auf Veränderung unterscheiden [5]. Beispielsweise sollten im vorintentionalen Stadium Nachteile einer Veränderung, in der Handlungsphase Vorteile einer Veränderung subjektiv stärker wahrgenommen werden; in der Phase der Absichtsbildung sollte Ambivalenz deutlich erlebt werden. Für Praktiker und Kliniker wesentlich ist die Annahme des Transtheoretischen Modells, dass das therapeutische Handeln den in den einzelnen Stadien unterschiedlichen Veränderungsprozessen (processes of change) bzw. motivationalen Ausgangsbedingungen angepasst sein sollte (stage matched interventions). Ein Patient, der noch im abwägenden Stadium der Absichtsbildung ist, wird von einer Konfrontationsübung nicht profitieren können, sondern muss sich erst für aktive Veränderungsschritte entscheiden; Motivational Interviewing wäre hier eine angemessene Gesprächsstrategie. Ein Patient, der sich bereits in der Handlungsphase befindet, erwartet hingegen von seinem Therapeuten Interventionsvorschläge und weniger ein „motivierendes Gespräch“.

\footnotetext{
${ }^{1}$ Die Tatsache, dass die im Hinblick auf motivationale Prozesse besonders wichtigen ersten beiden Stadien im Deutschen immer wieder divergierend übersetzt wurden, wird u.a. von Maurischat [4] diskutiert; dies lässt sich u. E. deshalb nicht auflösen, weil innerhalb der „Stadien“ heterogene psychologische Prozesse möglich sind (s.u.), was eine verbindliche Übersetzung erschwert.
}

Das Modell spielt besonders im Rahmen des Motivational-Interviewing-Ansatzes eine wichtige Rolle [6], da es betont, dass der eigentlichen Therapie wesentliche, motivierende Schritte vorausgehen müssen, die aus dem Rational der (Sucht-)Behandlung nicht einfach ausgeklammert werden können. Diese Meinung wird jeder Suchtbehandler teilen - über die empirische Bewährung des Modells ist damit aber noch keine Aussage getroffen!

Das Modell wurde ursprünglich an Rauchern entwickelt. Hierzu liegen auch die meisten Studien vor (vgl. [7]). Die Generalisierbarkeit dieses Stadienmodells auf andere Problemverhaltensweisen, einschließlich anderer Substanzabhängigkeiten und/oder psychischer Störungen, wird jedoch ausdrücklich postuliert. Es soll im Übrigen gleichermaßen für eigenständige oder therapeutisch geförderte Veränderungen gelten.

\section{Wie misst oder diagnostiziert man die Stadien?}

Wenn es für Behandlungserfolg und -vorgehen von entscheidender Bedeutung ist, in welchem Stadium sich Patienten befinden, dann ist es wichtig, dass Stadien ökonomisch, aber auch sicher und valide diagnostiziert werden könnten. Zur Messung der Veränderungsstadien haben sich ein kategorialer und ein dimensionaler Zugang entwickelt.

Im kategorialen Ansatz sind die Stadien durch bestimmte Einstellungen und Verhaltensweisen und bestimmte Zeitabschnitte operational definiert (stage algorithm): Wer zum Beispiel in den letzten 30 Tagen nicht intendiert hat, aufzuhören (mit Trinken oder Rauchen etc.), der ist dem Precontemplation-Stadium zuzuordnen (vgl. Tab.1).

Tab. 1 Beispiel für die operationale Definition der Veränderungsstadien bei Drogenabhängigen (stage algorithm, vgl. [13])

\begin{tabular}{|lll}
\hline Precontemplation & $\begin{array}{l}\text { Drogengebrauch während } \\
\text { des letzten Monats }\end{array}$ & $\begin{array}{l}\text { keine Absicht, in den } \\
\text { nächsten } 6 \text { Monaten auf- } \\
\text { zuhören }\end{array}$ \\
\hline Contemplation & $\begin{array}{l}\text { Drogengebrauch während } \\
\text { des letzten Monats }\end{array}$ & $\begin{array}{l}\text { Absicht, in den nächsten } \\
6 \text { Monaten aufzuhören, } \\
\text { aber nicht in den nächsten } \\
\text { 30 Tagen }\end{array}$ \\
Preparation & $\begin{array}{l}\text { Drogengebrauch während } \\
\text { des letzten Monats } \\
\text { Action }\end{array}$ & $\begin{array}{l}\text { Absicht, in den nächsten } \\
\text { kein Drogengebrauch } \\
\text { während des letzten } \\
\text { Monats, aber in den ver- }\end{array}$ \\
\hline gangenen 6 Monaten \\
kein Drogengebrauch \\
während der vergangenen \\
6 Monate
\end{tabular}

Abgesehen davon, dass in manchen Untersuchungen diese „Algorithmen“ nicht logisch konsistent formuliert wurden [8], stellt sich eine Reihe von Problemen: Zum einen sind die Zeitabschnitte, die zur operationalen Definition der Stadien herangezogen wurden, relativ willkürlich und variieren zwischen den Studien. Sutton [8] kritisiert deshalb, das zu messende Konstrukt sei eher 
als „planned time to action“ beschrieben und die Annahme, es handele sich um distinkte Stadien, sei nicht zu rechtfertigen. Wenn es aber keine deutlich unterschiedenen motivationalen „Stufen“ gebe, dann sei auch der Vorschlag jeweils spezieller motivierender Interventionen nicht gut begründet. Die Kritik an dem Algorithmenansatz lässt sich sogar noch fortsetzen: Es handelt sich nämlich im Kern um einen Prototypen- oder Extremgruppenansatz, in dem viele Patienten sich nicht abbilden lassen. Insbesondere gilt dies für Betroffene mit einem inkonsistenten Konsum, häufigen Rückfällen oder „kontrollierten“ Konsummustern. (Dies kann man an dem Beispielalgorithmus (Tab.1) durchspielen.) Die gewählten Kategorien bilden auch die vorhandene Heterogenität von Patientengruppen in keiner Weise ab: Alle Patienten einer Alkoholentwöhnungstherapie etwa unternehmen im Prinzip etwas gegen ihre Abhängigkeit, allein schon durch die Teilnahme; sie müssten einheitlich dem Handlungsstadium zugeordnet werden - die in Wirklichkeit von Therapeuten beobachteten großen Motivationsunterschiede innerhalb dieser Gruppe werden hier nivelliert und es lassen sich aus dem Modell keine ausreichend differenziellen Vorhersagen ableiten.

Nicht zuletzt aufgrund dieser Probleme wurden auch dimensional konzipierte Fragebogenansätze entwickelt. Die wichtigsten sind die

- University of Rhode Island Change Assessment Scale (URICA [9]); die

- Stages of Change Readiness and Treatment Eagerness Scale (SOCRATES [10]); und die

- Readiness to Change Questionnaire (RCQ [11]),

die allesamt auch in deutschsprachigen Versionen vorliegen (vgl. im Überblick [7] und das Elektronische Handbuch für Erhebungsinstrumente im Suchtbereich). Gemeinsam ist diesen Ansätzen, dass die für die Stadien jeweils typischen Einstellungen oder verhaltensbezogenen Selbstaussagen als Fragebogenitems vorgegeben werden (vgl. Tab. 2).

Tab. 2 Beispielitems aus der Veränderungsstadienskala (deutsche Fassung der URICA, vgl. [12])*

\begin{tabular}{ll}
\hline Precontemplation & $\begin{array}{l}\text { Ich vermute, ich habe Schwächen, aber es gibt nichts, } \\
\text { was ich wirklich verändern müsste. }\end{array}$ \\
Contemplation & $\begin{array}{l}\text { Ich habe ein Problem, und ich denke wirklich, dass ich } \\
\text { daran arbeiten sollte. }\end{array}$ \\
\hline Action & $\begin{array}{l}\text { Ich arbeite wirklich hart daran, mich zu verändern. } \\
\text { Maintenance }\end{array}$ \\
$\begin{array}{l}\text { Das, was ich schon geändert habe, ist für mich nicht } \\
\text { so leicht beizubehalten, wie ich gehofft hatte, und ich } \\
\text { bin hier, um einen Rückfall zu verhindern. }\end{array}$
\end{tabular}

* Preparation wird mit diesem Fragebogen nicht erfasst.

Fragebogenverfahren werfen jedoch ebenfalls erhebliche methodische Probleme auf. Vor allem lassen sich aufgrund des jeweils gefundenen Messwerteprofils nicht ohne weiteres Zuordnungen zu bestimmten Stadien begründen.

Studien mit psychometrisch konstruierten Skalen zeigen in der Regel hohe Korrelationen zwischen einzelnen stadienbezogenen Subskalen [7], was der Annahme, es handele sich um distinkte Stadien, widerspricht. Clusteranalysen erbrachten keine für die postulierten Stadien spezifischen Strukturen, sondern eine verwirrende Vielzahl unterschiedlicher Cluster, die zum Teil unsystematische Erhöhungen mehrerer Subskalen aufwiesen und demnach im Sinne des Modells kaum sinnvoll interpretierbar sind.

Insgesamt legen die Arbeiten zu URICA, SOCRATES und RCQ den Schluss nahe, dass eine mehrdimensionale Konzeption der Veränderungsmotivation mit kontinuierlichen Skalen psychometrisch befriedigender sein dürfte als Versuche, mittels Clusteranalysen (z.B. [5]) oder anderen Methoden [11] scheinbar diskrete Stadien zu generieren. Bei Verwendung interkorrelierter mehrdimensionaler Skalen bleibt allerdings streng genommen das Modell auf der Strecke und der pragmatische Gebrauch nach dem Motto „Patient x (z. B. Contemplation) braucht Inter-

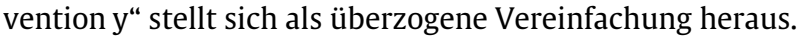

Zur konvergenten Validität kategorialer Algorithmen und psychometrischer Instrumente existieren nur wenige Studien, die insgesamt Zweifel an der konvergenten Validität kategorialer Algorithmen und psychometrisch konstruierter Instrumente wecken $[13,14]$.

\section{Empirische Bestätigung und kritische Bewertung}

Wenn die Messinstrumente weitreichende Probleme aufwerfen, dann stellt sich die Frage nach der empirischen Bestätigung des Modells umso dringlicher.

Die weitaus meisten Studien zu den Stadien der Veränderung sind querschnittlich angelegt und dienen der Untersuchung von Korrelaten der Veränderungsstadien (vgl. [8]). Solche Untersuchungen, die mit einem Stadienalgorithmus Extremgruppen untersuchen und dann zum Beispiel herausfinden, dass - oft hochselegierte - Probanden im Precontemplation-Stadium und im Handlungsstadium sich in ihren Einstellungen zu einem Problemverhalten (oder anderen Merkmalen) unterscheiden, liefern durchaus einen notwendigen Beitrag zur Konstruktvalidität des Verfahrens; sie beruhen aber auf wissenschaftstheoretisch gesehen wenig „strengen“ Prüfungen und sind im Hinblick auf die eigentlichen Vorhersagen des Modells nicht hinreichend. Kriterienbezogene Fragen nämlich etwa, ob das Modell auch einen Anwendungsnutzen im Sinne von Vorhersage und selektiver Indikation von Maßnahmen hat, werden dadurch nicht beantwortet. Durch dieses Missverhältnis zwischen der Menge erfolgreicher „Tests“ des Modells und dem Mangel an wirklich strengen Prüfungen ist u. E. die manchmal anzutreffende Überschätzung des Modells zu verstehen. Die Zuordnung von Interventionen auf der Basis des Modells ist jedenfalls nicht aus querschnittlichen Befunden ableitbar und die propagierte breite Anwendung ist nur intuitiv, nicht aber empirisch legitimiert. Dazu müsste das Modell die Entwicklung einzelner Individuen vorhersagen können und es müsste prognostischen Wert in der Prädiktion von Therapieerfolg, Rückfallquote und bezüglich der Passung von Therapeut und Patient beweisen. Sutton [8] fordert deshalb zu Recht, dass neben querschnittlichen Designs

1. Studien zu der vorhergesagten Abfolge der Stadien,

2. longitudinale Studien zur Vorhersage des Wechsels von einem Stadium zum anderen sowie 
3. feldexperimentelle Studien zur Passung bzw. Nichtpassung zwischen Patientenstadium und therapeutischen Interventionen

durchgeführt werden müssten.

Aber, so Sutton: „No published studies on alcohol or drug use could be found that used any of the last three types of research design“ (p. 180). Die begeisterte Rezeption des Modells ist demnach eher auf seine pragmatischen und einfachen Konzepte als auf wirkliche empirische Bewährung zurückzuführen.

In einer aktuellen eigenen Studie bei Alkoholpatienten in stationärer Entwöhnungstherapie [15] haben wir neben anderen Fragen untersucht, ob das Veränderungsstadium Vorhersagewert für die Abbruchquote in der Therapie, für die Therapiedauer und für die Rückfallrate hat. Dies war auf der Grundlage der Selbstaussagen der Teilnehmer in der Veränderungsstadienskala für keine der vorherzusagenden Variablen der Fall. Ob dies auf der Grundlage von Therapeutenaussagen besser gelingt, wird derzeit analysiert.

Ein weiterer Befund dieser Studie: Eine Clusteranalyse ergab als am besten interpretierbare Lösung nicht die im Modell vorgesehenen vier, sondern drei Cluster. Neben einem modellkonformen „Precontemplation-Typ“ (in der Therapie!) mit nur auf der Precontemplation-Skala erhöhten Werten zeigte sich auch ein „Veränderungstyp“, bei dem Absicht und Handlung sich aber nicht differenzieren lassen (hohe Werte bei Absichtsbildung und Handlung). Ein drittes Cluster war durch niedrige Werte auf allen Veränderungsskalen gekennzeichnet. Also: keine Leugnung des Problems (= niedrige Precontemplation-Werte), aber auch keine starke Intention zur Veränderung. Dieses - nach dem Modell nicht zu erwartende - Muster benannten wir analog zu DiClemente und Hughes [5] als eines von „uninvolvierten“ Patienten. Aus der klinischen Erfahrung lässt sich dieses Cluster durchaus gut einordnen: Es beschreibt Patienten, die sich zwar aus meist extrinsischer Motivation in der Therapie befinden und dort auch „mitmachen“, jedoch ohne dass dies ein hohes Involvement und echte Eigenverantwortlichkeit impliziert. Dies ist eine unter Versorgungsgesichtspunkten hoch interessante Gruppe, und die therapeutische Aufgabe, bei diesen Patienten Motivationsarbeit zu leisten, ist sicher nicht leichter als bei jenen, die ihr Problem leugnen. Wichtig ist hier die Frage der Wahl der Intervention: Tendenziell sind bei uninvolvierten Patienten andere Gesprächstechniken und Interventionsangebote als effektiv anzusehen. Problematisch ist aber, dass die Erforschung der Frage, welche dies sein mögen (vermutlich sehr strukturierte Angebote), durch das Stadienmodell nicht erleichtert wird, weil in ihm eine Einordnung dieser Patienten überhaupt nicht möglich ist.

Bei der Beschäftigung mit dem Modell im Suchtbereich, insbesondere bei alkohol- und drogenbezogenen Störungen, stellen sich darüber hinaus bereits aufgrund klinisch-sachlogischer Überlegungen kritische Fragen. Insbesondere: Ist die Grundannahme plausibel, dass man die Veränderungsbereitschaft im Hinblick auf alle gesundheitsbezogenen Verhaltensweisen wirklich wissenschaftich befriedigend mit einem (simplen) Schema beschreiben kann? Ist es psychologisch und körperlich wirklich der gleiche Veränderungsprozess, ob jemand an einem Tag kein
Problembewusstsein bezüglich seiner Zahngesundheit hat, am nächsten Tag Zahnschmerzen bekommt (Absichtsbildung) und am dritten Tag den Zahnarzt aufsucht (Handlungsstadium; beliebige andere Beispiele sind möglich) - oder ob er nach mehreren Jahrzehnten einer Suchtkarriere in der soundsovielten Einrichtung eine konkrete Maßnahme beginnt?

Weitere Probleme, die sich gerade bei langjährigen Suchtpatienten stellen, sind die Komorbidität und die Demoralisierung.

Wohl wenige Suchtpatienten haben nur ein Problem (auch wenn es selbstwertdienlich und aus der Patientenperspektive verständlich ist, dies so zu sehen). Die meisten haben auch berufliche, soziale oder familiäre Probleme. Häufig bestehen aber auch komorbide psychische Störungen. Was bedeutet es für den Veränderungsprozess, wenn der Patient (noch) nicht für alle relevanten Probleme gleichermaßen Veränderungsintentionen hat? Patienten, die für all ihre Probleme im Handlungsstadium sind, weisen kardinal andere motivationale Voraussetzungen auf als solche, die nur ihr Suchtproblem angehen wollen. Dies unterstreicht, wie reduktiv die Information: „Person $\mathrm{x}$ ist im Handlungsstadium“ sein kann.

Die Tatsache der für viele langjährige Suchtpatienten typischen Demoralisierung ist ebenfalls zu bedenken; sie wird durch den Terminus „Precontemplation“ sicher nicht beschrieben. Hier gilt eher die aus dem Dosis-Effekt-Modell, einem anderen einflussreichen „Stadienmodell“ (vgl. [17]), entlehnte Regel, dass ein Patient erst aus der Demoralisierung „heraus muss“, bevor die „Remoralisierung“ beginnen kann. Psychosoziale Hilfsmaßnahmen im weitesten Sinne [18], beginnend mit der Alkohol- oder Drogenentzugsbehandlung, wären hier indiziert. Dabei gilt der Hinweis, dass sich die Hilfeangebote und -wege auch zwischen den verschiedenen Substanzabhängigkeiten deutlich unterscheiden und Angebote wie zum Beispiel die Substitutionsbehandlung nur im Bereich der Heroinabhängigkeit ernsthaft diskutiert werden. Auch dies ist ein weiterer Beleg dafür, dass das Stadienmodell nicht undifferenziert auf den Suchtbereich übertragen werden kann.

Fassen wir die genannten empirischen Ergebnisse und die formulierte sachlogische Kritik zusammen, so kann man dem Stadienmodell der Veränderung einen heuristischen Wert für Orientierung hinsichtlich der Veränderungen und der mit ihnen verbundenen Prozesse zwar durchaus zugestehen; dass es eine empirisch bestätigte Lösung für konkrete theoretische oder anwendungsbezogene Probleme im Suchtbereich darstellt, kann bei kritischer Betrachtung aber noch keineswegs behauptet werden.

Neue Entwicklungen und Lösungsansätze: das Rüickfallstadium

Angesichts der geringen empirische Bewährung des Modells im Bereich von Alkholabhängigkeit und Drogenkonsum erscheint der Vorschlag von Sutton [8], hier neue oder andere Stadienmodelle zu entwickeln, verständlich. Da sich das ursprüngliche Modell bereits ausgezeichnet etabliert hat (s. o.), sollten Weiterentwicklungen unserer Auffassung nach auf diesem aufbauen und es als Orientierungsrahmen heranziehen. 
Im Folgenden möchten wir deshalb ein revidiertes Modell vorschlagen, das die besondere Rolle von Rückfällen und chronischen Verläufen im Veränderungsprozess bei substanzbezogenen Störungen berücksichtigt, ohne Kernüberlegungen und -konzepte des Veränderungsmodells aufzugeben. Eine wichtige Erwartung, die dieses Modell erfüllen sollte, ist, dass Personen mit substanzbezogenen Störungen spezifischer beschrieben werden können und dass spezifischere therapeutische Handlungsanweisungen ableitbar sind. Es sollen nicht nur besonders „prägnante“ Stadien erfasst werden, sondern auch und gerade der für lange Verläufe im Suchtbereich typische Zwischenbereich.

Wir können dabei auf einen früheren Vorschlag des Canadian Fitness and Lifestyle Research Institute zurückgreifen [19]. Grundlegend ist der Gedanke, dass (aktuelle und frühere) „Aktivitäten" und Intentionen grundsätzlich unabhängige Konstrukte sind. Intentionen machen Handlungen wahrscheinlicher, ob Handlungen aber wirklich vollzogen und vor allem auf Dauer vollzogen werden, ist von zusätzlichen volitionalen Prozessen abhängig.

Patienten, die zwar während einer Phase des Rückfalls keine sichtbaren Veränderungsbemühungen unternehmen, bei denen die Intention zur Abstinenz aber prinzipiell besteht, wären mit dem bisherigen Stadienmodell nicht einzuordnen. Dabei sind sie gerade in der Suchtversorgung eine wichtige Gruppe. Unterscheidet man nach Intention, früherer Handlung und aktueller Handlung, so ergeben sich rein logisch drei zusätzliche Stadien, die die angesprochenen Probleme modellhaft abbilden können: „Rückfall“, „Rückfallgefahr“ und „Resignation/Dropout“ (vgl. Tab.3). Beispiele sollen die drei zusätzlichen Stadien verdeutlichen:

Rückfall: Ein Patient mit einem Rückfall (relapse, nicht lapse) unterbricht seine Veränderungsmaßnahmen und ist deswegen nicht dem Aufrechterhaltungsstadium zuzuordnen. Dass er kein Problembewusstsein hat, ist möglich (Precontemplation), aber keineswegs selbstverständlich. Alle Rückfälligen, die grundsätzlich abstinent leben möchten, dies aber aufgrund verschiedener Defizite (hinsichtlich psychosozialer Variablen wie Abstinenzzuversicht, Selbstmanagementfähigkeiten, Selbstkontrolle, psychiatrischer Komorbidität etc.) oder Umgebungsvariablen nicht erreichen können, sind hier einzuordnen.

Rückfallgefahr: Patienten, die zwar auf äußeren Druck oder um anderen etwas zu beweisen, an Maßnahmen erfolgreich teilnehmen, deren Intentionen aber langfristig nicht am Abstinenzziel ausgerichtet sind, fallen in diese Gruppe.
Resignation/Drop-out: Patienten, die demoralisiert sind und mehrere langfristig erfolglose Abstinenzversuche hinter sich haben, geben ihre Veränderungsbemühungen schließlich auf. Dies muss ähnlich wie im Stadium des Rückfalls aber nicht mit mangelndem Problembewusstsein zu tun haben, vielmehr wird das Suchtverhalten trotz des Wissens um seine selbstschädigenden Konsequenzen fortgesetzt.

Den Stadien „Rückfall“ und „Resignation“ ist gemeinsam, dass die motivationalen Voraussetzungen für die Anwendung bewältigungsorientierter Interventionen zunächst fehlen: Gesprächsstrategien, denen das Prinzip des Motivational Interviewing zugrunde liegt, erscheinen demgegenüber indiziert. Der Akzent in der Gesprächsführung unterscheidet sich deutlich von dem bei Patienten, die eine weniger lange Vorgeschichte aufweisen (die also wirklich noch im Precontemplation-Stadium sind). Allgemeine Informationsvermittlung über die Sucht ist möglicherweise nicht (mehr) indiziert und es können im Beratungsgespräch auslösende und aufrechterhaltende Bedingungen nicht nur in Abhängigkeitsphasen, sondern auch für Abstinenzphasen exploriert werden.

Aus diesem revidierten Modell lassen sich erweiterte und spezifischere Gesprächstechniken für Therapeuten und Berater ableiten. Alkoholiker zur Abstinenz zu motivieren, die bereits erfolgreiche Abstinenzphasen erlebt haben, kann (muss aber nicht) im Sinne eines ressourcenaktivierenden Vorgehens leichter sein, weil hier an frühere Erfolgserlebnisse appelliert werden und dies die Selbstwirksamkeitserwartung steigern kann.

Für Patienten aus dem Resignation/Drop-out-Stadium sind psychotherapeutische Interventionen im engeren Sinne hingegen zunächst überhaupt nicht indiziert (weil nicht aussichtsreich), sondern eher psychosoziale Maßnahmen im Sinne der Schadensminimierung erforderlich (vgl. [18]).

\section{Ausblick}

Aus dem hier skizzierten, spezifisch auf den Suchtbereich zugeschnittenen Modell sollten präzisere Vorhersagen ableitbar sein und es sollte sich erfolgreich empirischer Prüfung stellen können.

In diesem Zusammenhang ist jedoch von vornherein einzuräumen, dass auch diesem Modell einige Schwächen des Originalansatzes inhärent sind: So wären die Stadien grundsätzlich nicht in der vorliegenden Skizze, aber im Rahmen zukünftiger Forschung - genauer zu definieren. Dabei sollten nicht wie bis-

\begin{tabular}{llll}
\hline & frühere Aktivitäten & jetzige Aktivitäten & Veränderungsintention \\
\hline Precontemplation & nein & nein & nein \\
\hline Absichtsbildung/Vorbereitung & nein & nein & ja \\
\hline Handlung & nein & ja & ja \\
\hline Aufrechterhaltung & ja & ja & ja \\
\hline Rückfall & ja & nein & ja \\
\hline Rückfallgefahr & ja/nein & ja & nein \\
\hline Resignation/Dropout & ja & nein & nein \\
\hline
\end{tabular}

Tab. 3 Stadien der Veränderung und Rückfallgeschehen: ein spezifisches dem Rückfallgeschehen im Suchtbereich angepasstes Stadienmodell 
her einzelne Stadien eher durch Einstellungen (Precontemplation, Contemplation, Rückfallgefahr), andere eher durch Verhalten (Handlung, Aufrechterhaltung, Rückfall) beschrieben werden (vgl. [4]), sondern alle Stadien sollten vollständiger als bisher durch für sie typische Einstellungen und Verhaltensweisen, ggf. auch durch die vorliegenden Kompetenzen (Resignation), beschrieben werden. Die Hinzunahme solcher Dimensionen, die Stadien beschreiben, würde Vorhersagen präziser machen, das Modell wäre aber auch schwerer überschaubar und seine bisherige Stärke der Einfachheit reduziert.

Die kritischen Abschlussbemerkungen sollen eines verdeutlichen und unterstreichen: Trotz seiner immensen Bedeutung ist das Stadienmodell der Veränderung keine anbetungswürdige Sache, sondern nicht mehr als ein Orientierungsmodell, das in Zukunft viel stärker als bisher der natürlichen wissenschaftlichen „Evolution“ ausgesetzt sein sollte.

\section{Literatur}

${ }^{1}$ Heidenreich T, Hoyer J. Stadien der Veränderung in der Psychotherapie: Modelle, Perspektiven, Kritik. Verhaltenstherapie \& psychosoziale Praxis 1998; 30: $381-402$

2 Prochaska JO, DiClemente CC, Norcross JC. In search of how people change. American Psychologist 1992; 47: 1102 - 1114

${ }^{3}$ Keller S, Wayne WV, Prochaska JO. Das Transtheoretische Modell Eine Übersicht. In: Keller S (Hrsg). Motivation zur Verhaltensänderung. Das Transtheoretische Modell in Forschung und Praxis. Freiburg: Lambertus, 1999: 17- 44

${ }^{4}$ Maurischat C. Erfassung der „Stages of Change“ im Transtheoretischen Modell - eine Übersicht dimensionaler versus kategorialer Ansätze. Zeitschrift für Klinische Psychologie, Psychiatrie und Psychotherapie 2002; 4: $343-367$

${ }^{5}$ DiClemente CC, Hughes SO. Stages of change profiles in outpatient alcoholism treatment. Journal of Substance Abuse 1990; 2: 217-235

${ }^{6}$ DiClemente C, Velasquez MM. Motivational interviewing and the Stages of Change. In: Miller WR, Rollnick S (Hrsg). Motivational Interviewing: Preparing people for change. New York: Guilford, 2002: 201-216
${ }^{7}$ Heidenreich T, Hoyer J. Stadien der Veränderung bei Substanzmissbrauch und -abhängigkeit: eine methodenkritische Übersicht. Sucht 2001; 47: $158-170$

${ }^{8}$ Sutton S. Back to the drawing board? A review of applications of the transtheoretical model to substance use. Addiction 2001; 96: $175-186$

${ }^{9}$ McConnaughy EA, Prochaska JO, Velicer WF. Stages of change in psychotherapy: Measurement and sample profiles. Psychotherapy 1983; 20: $368-375$

${ }^{10}$ Miller WR, Tonigan JS. Assessing drinkers' motivation for change: The Stages of Change Readiness and Treatment Eagerness Scale (SOCRATES). Psychology of Addictive Behaviors 1996; 10: 81 - 89

${ }^{11}$ Rollnick S, Heather N, Gold R et al. Development of a short „readiness to change" questionnaire for use in brief, opportunistic interventions among excessive drinkers. British Journal of Addiction 1992; 87: $743-754$

${ }^{12}$ Heidenreich T, Hoyer J, Fecht J. Veränderungsstadienskala. In: Rist F, Küfner H, Glöckner-Rist A et al (Hrsg). Elektronisches Handbuch für Erhebungsinstrumente im Suchtbereich. Mannheim: Zentrum für Umfragen, Methoden und Analysen 2001; Version 1.00

${ }^{13}$ Belding MA, Iguchi MY, Lamb RJ. Stages of change in methadone maintenance: Assessing the convergent validity of two measures. Psychology of Addictive Behaviors 1996; 10: 157-166

${ }^{14}$ Hodgins DC. Stages of change assessments in alcohol problems: Agreement across self- and clinician-reports. Substance Abuse 2001; 22: 87-96

${ }^{15}$ Hoyer J, Heidenreich T, Fecht J et al. Stadien der Veränderung in der stationären Alkoholentwöhnungstherapie. Verhaltenstherapie 2003; 13: $31-39$

${ }^{16}$ Timko C, Moos RH, Finney JW. Long-term outcomes of alcohol use disorders: Comparing untreated individuals with those in Alcoholics Anonymous and formal treatment. Journal of Studies on Alcohol 2000; 61: 529-540

${ }^{17}$ Lueger RJ. Ein Phasenmodell der Veränderung in der Psychotherapie. Psychotherapeut 1995; 40: 267-278

${ }^{18}$ Denning P. Practising harm reduction therapy: An alternative approach to addictions. New York: Guilford, 2000

${ }^{19}$ Canadian Fitness and Lifestyle Research Institute. Stages of change in exercise. The Research File 1995; 95-05 